\title{
I Congreso esPañol de Filosofía Práctica o APLICADA FILOSOFÍA Y ESPACIOS SOCIALES
}

\author{
Ist SPANiSh Conference on PHILOSOPHICAL PRACTICE \\ PHILOSOPHY AND SOCIAL PLACES
}

\author{
JoAn MÉndez CAMARASA \\ Presidente de la Asociació de Filosofía Pràctica de Catalunya \\ Universidad de Barcelona \\ jmendez@afpc.es
}

RECIBIDO: 9 DE DICIEMBRE DE 2011

ACEPTADO: 22 DE DICIEMBRE DE 2011

\begin{abstract}
Resumen: Del 18 al 20 de abril de 2011 se celebró en Barcelona el I Congreso Español de Filosofía Práctica o Aplicada. La organización del evento fue a cargo de la Asociación de Filosofía Práctica de Cataluña (afpc). El I Congreso Nacional de Filosofía Práctica o Aplicada del estado español ha querido mostrar de qué manera y en qué ámbitos los profesionales de la filosofía están desarrollando actividades vinculadas con su formación. Convencidos de que no cabe reducir (ni confundir) la práctica de la filosofía con el trabajo docente que se efectúa en los centros de enseñanza, ya sean éstos escolares o universitarios, desea dar a conocer distintas experiencias que se están llevando a cabo en cuanto a la aplicación de los conocimientos y las herramientas propias de la filosofía en la actualidad, para intervenir en diferentes espacios sociales aportando reflexiones y soluciones a los problemas que se plantean
\end{abstract}

Palabras clave: Asesor filosófico, filosofía aplicada, diálogo socrático, filosofía y empresa, Internet

\begin{abstract}
First Spanish Conference on Philosophical Practice has been celebrated in Barcelona from $18^{\text {th }}$ to $20^{\text {th }}$ April (2011). This conference was organized by Catalonian Philosophical Practice Association. Its aim was showing where and how experts on philosophy are carrying out activities connected to their training. Taken for granted that philosophical practice shouldn't be restricted nor confused with teaching philosophy at schools and universities, this conference wanted to show the wide variety of activities and projects that are being accomplished in this field today. By means of it, Philosophical Practice wants to implement its knowledge and its tools inside different areas and to provide thought and solutions to problems that might appear in different social spheres
\end{abstract}

Keywords: Philosophical counselor, Philosophical Practice, socratic dialogue, Philosophy in Business, Internet 


\section{Introducción}

Una vez contabilizados ya diez congresos internacionales ${ }^{1}$ y un congreso iberoamericano en Filosofía Aplicada, desde la Asociación de Filosofía Práctica de Cataluña (afpc) creímos que había llegado el momento de empezar a movilizarse para organizar el primer congreso nacional, que permitiera reunir una buena representación de los filósofos del Estado español que nos encontramos trabajando en dicha dirección. Con esta intención, y una vez aprobado el proyecto en la asamblea anual de la afpc, se creó la web: www.congreso.afpc.es, que serviría para ofrecer toda la información necesaria: cuándo se iba a llevar a cabo, dónde, de qué manera se debían presentar las comunicaciones, talleres, plazos de entrega, etc. La publicación de dípticos y la difusión por internet fueron el método empleado para dar a conocer el evento de manera amplia.

Asimismo, nos pusimos en contacto con el Dr. José Barrientos, quien generosamente se ofreció desde el principio para ofrecernos la ayuda que necesitáramos, dada su experiencia en la organización del I Congreso Iberoamericano y el VIII Congreso Internacional. Igualmente, desde el grupo de investigación "Filosofía Aplicada. Sujeto, sufrimiento, sociedad" (HUM 018) y del grupo ETOR ${ }^{2}$ asumirán el compromiso de la publicación de las actas ${ }^{3}$. El primer paso estaba dado.

\section{Filosofía y espacios sociales}

Si algo tenemos en común como mínimo quienes compartimos la inquietud por la filosofía práctica es, sin lugar a dudas, la de reconocer la

\footnotetext{
${ }^{1}$ Se está preparando ya el undécimo congreso internacional, que tendrá lugar en julio del 2012 en la República de Corea. Para más información se puede visitar la web: http://www.icpp10.org/

${ }^{2}$ Las siglas de ETOR se corresponden con: educación y tratamiento de orientación racional.

${ }^{3}$ Dichas actas ya han sido publicadas. Dado el volumen del material, se han repartido en sedas publicaciones: BARRIENTOS, José, y MÉNDEZ, Joan (editores), Filosofía y espacios sociales, e Intervenciones filosóficas en la sociedad, Visión Libros, Madrid, 2011. La presentación de ambos libros tuvo lugar en la librería Catalonia el 5 de noviembre de 2011, por parte de ambos editores. Una reseña de dichas publicaciones se pueden leer en esta misma revista en el apartado de Sección bibliográfica.
} 
necesidad de hacer salir a la filosofía "de la caverna". Convencidos de su utilidad y de los beneficios públicos y privados que ésta puede proporcionar, tratamos de llevar la filosofía allá donde no se la espera, colonizando así nuevos espacios en los que cabe esperar que su aparición muestre su relevancia. Es por ello que dedicamos dicho título al congreso, toda una declaración de intenciones.

De esta manera, los ejes temáticos sobre los que girarían las exposiciones que se presentaran serían los siguientes:

- El despacho del asesor filosófico y la atención personalizada.

- El filósofo en la empresa.

- Los cafés y talleres filosóficos.

- Filosofía interdisciplinar: música, fotografía, teatro, cine, ciencia, literatura...

- Los centros culturales y museos como espacios de divulgación.

- Filosofía para niños.

- Filosofía para grupos y organizaciones: cárceles, hospitales, comités de bioética...

- Turismo filosófico.

- Internet, radio y televisión como espacios para la filosofía.

\section{El congreso}

Una vez finalizado el trabajo de selección del abundante material que fuimos recibiendo en el plazo asignado, llegó el momento de la realización efectiva del congreso. Las fechas serían el 18, 19 y 20 de abril, y la sede el colegio salesiano San Juan Bosco de Barcelona, cuyo director titular, don Ramón Vera, tuvo la amabilidad de poner a nuestra disposición todas sus instalaciones: aulas, sala de audiovisuales, teatro, etc. El congreso se desarrollaría en sesiones de mañana y tarde durante los tres días. Sin duda, el horario se presentaba muy apretado, pero se estimó que valía la pena favorecer que todo el mundo pudiera asistir a todos los actos, evitando así simultanear sesiones y obligar a que uno tuviera que elegir a cuál asistir, cuando quizá ambas eran de su agrado. 
El lunes 18 se comenzó a las $9 \mathrm{~h}$. con el reparto de las acreditaciones y la entrega del material correspondiente. El número de personas acreditadas y que fueron siguiendo las jornadas fue aproximadamente de 70 personas. Tras el acto de apertura, efectuado por la Junta de la afpc, continuamos con la exposición de Luisa López Cabrejas acerca de las dinámicas de los cafés filosóficos, en los que posee ya una experiencia contrastada. Tras el descanso, José Barrientos nos habló de los intercambios online como marco de desarrollo de la Filosofía Aplicada a la Persona, destacando así las posibilidades que ofrece internet para el asesoramiento filosófico, y de qué manera podemos potenciar las ventajas de dicho medio, así como qué estrategias podemos emplear para minimizar sus inconvenientes.

Seguirían las comunicaciones: Miquel Martínez sobre la filosofía como cartografía, Eduardo Vergara expondría su labor profesional como filósofo en centros penitenciarios, y María Tardón elaboraría un discurso sobre el concepto de Reconocimiento en las instituciones, dentro del ámbito de la ética aplicada.

Por la tarde, Jaume Puigferrat presentó una polémica ponencia sobre "Cómo ganar dinero con la filosofía". Nos habló de la necesidad de adoptar una mentalidad empresarial a la hora de ofrecer servicios filosóficos de carácter práctico, algo de lo que según su punto de vista tienden a carecer nuestros licenciados en filosofía. Las comunicaciones que vendrían a continuación serían las de Miquel Comas, que trataría los límites y contradicciones observables en las críticas de WikiLeaks a las "tiranías empresariales"; la de Óliver Álvarez sobre cómo salir al ágora en el siglo XXI; Francisco Javier López Frías y su reivindicación de una ética del deporte; y Miguel Mandujano, quien relacionaría la Acción y el clinamen en el Proyecto de Vida como orientación filosófica.

Dos talleres sirvieron para finalizar este primer día de congreso: el de Miquel Pardo, dedicado a la fenomenología del sentimiento estético en la práctica filosófica; y el de Artur Massana y Vander Lemes, acerca de cómo determinados espacios arquitectónicos pueden ser un excelente punto de arranque para propiciar la reflexión filosófica.

El martes 19 se inició con dos lecturas dramatizadas en el teatro. Los textos escogidos fueron: Adán y Eva: ave o nada, lectura retrospectiva 
del pecado original, y Übermensch, el superhombre en la era digital ${ }^{4}$. Las personas que se encargaron de la dramatización fueron Mónica Planchat, Carles Pascual, Ana Galisteo y Joan Méndez.

A continuación, y ya en la sala de audiovisuales, se procedió a la exposición de "La Fiesta de la Filosofía", por parte de Juan Carlos Gómez y Joan Méndez. El nombre de la sesión hacía referencia a la iniciativa de la mayor parte de los centros cívicos de Barcelona de organizar todos ellos en la misma semana actividades diversas relacionadas con la filosofía. La conferencia sirvió para conocer cuáles fueron las dinámicas que se llevaron a cabo en cada uno de ellos y la acogida que habían tenido 5 .

Tras el descanso, Anna Sarsanedas hablaría sobre Filosofía e Internet, para hacernos ver las múltiples opciones que nos ofrece la red en el ámbito de la filosofía. Siguieron las comunicaciones de: Erika Adánez y José Luis Romero, quienes expusieron su proyecto en Alter consulta, con la colaboración interdisciplinar entre filosofía y psicología; Soledad Hernández, planteando cómo los espacios de reinserción social y laboral pueden ser también espacios para la filosofía; Iñaki Martínez, quien nos obsequió diversas consideraciones en torno a la vergüenza de ser hombre; y Teresa Gaztelu, que abordó el sufrimiento desde el asesoramiento filosófico y el budismo.

La primera conferencia de la tarde iría a cargo de Begoña Román, que se centraría en la ética en y de las organizaciones: experiencias buenas y malas. Mercè Blasco nos habló en su comunicación sobre el Proyecto Bona Vida 099, Ana Bohoyo nos introdujo en la figura del filósofo como agente moral en el campo de la bioética, mientras que Lucas Sanou abordó el tema de las microexpresiones y la comunicación no verbal, como aspectos que debe saber interpretar el asesor filosófico para detectar cuándo el discurso hablado y el lenguaje corporal presentan incoherencias.

\footnotetext{
4 Ambas son obras originales de Joan Méndez, que pueden encontrarse en: http://filomendez.blogia.com

${ }^{5} \mathrm{La}$ afpc colaboró con el evento, a petición de los centros cívicos casa elizalde y golferichs de Barcelona, tanto en su difusión -se puso una carpa cerca de Plaza Cataluña con lectura de textos filosóficos para invitar a la participación- como en su misma realización -organizando cafés filósoficos, lecturas dramatizadas, cinefòrum, etc.
} 
Los tres talleres que cerraron la sesión del martes fueron el de Henrik Hernández, sobre la angustia creadora en psicoterapia; de nuevo Artur Massana y Vander Lemes, en este caso sobre los diálogos neosocráticos en la empresa; y el de Iván Redondo y Rubén Carreño, quienes presentaban la posibilidad de efectuar una reflexión filosófica a través del diálogo en un juego de rol.

El miércoles 20 Pepe Ordóñez se encargó de abrir la sesión con una conferencia de título asombroso: "Anarco-existencialismo: una visión ontológica de la resignificación subjetiva". Tras el descanso, continuamos con Irene de Puig, quien se preguntaba si puede ayudar la filosofía a prevenir la violencia. Las comunicaciones siguientes fueron a cargo de: Toni Mañach, quien se planteaba la función ética en el diseño como una forma de filosofía aplicada; Raúl Francisco Sebastián, que abordó la responsabilidad social de la empresa deportiva; y Nacho Bañeras, quien presentó el asesoramiento filosófico como tarea crítica.

Por la tarde, Mercedes García Márquez nos habló de la filosofía a la intemperie, en clara alusión a una filosofía que sale de la caverna y se expone ante todos y para todos. Las comunicaciones que sirvieron de antesala al acto de clausura fueron a cargo de: Albert Llorca, que trató la relación entre filosofía y turismo; Humbert Ruiz, quien destacó el papel del filósofo en el ámbito empresarial; Víctor Páramo, sobre bioética intercultural y filosofía de la conciencia; y por último, Mariano Fernández, que abordó el papel de la forma en el espacio, esto es, la conexión íntima entre escultura y pensamiento, a la vez que relacionó todo ello con su afición al senderismo.

\section{Acto de clausura}

Joan Méndez, como presidente de la asociación organizadora (afpc) dio las gracias a todos los ponentes, comunicantes y asistentes en general por su participación entusiasta y su colaboración en estas jornadas. Valoró muy positivamente el nivel de las intervenciones, y, por encima de todo, la oportunidad de celebrar un congreso a nivel estatal español que permitiera reunir una importante representación del trabajo que se está llevando a cabo en el país. Otras personas relevantes en este campo, 
como Mónica Cavallé, excusaron no poder asistir al evento por tener compromisos de agenda previos, pero nos consta su voluntad de colaborar en futuros encuentros. Confiamos en que no tardemos en ver cómo alguien recoge el testigo de Barcelona y se propone organizar una nueva cita que permita poner en común de nuevo los esfuerzos de unos y otros.

También hubo palabras de agradecimiento para Ana Galisteo, Joan Carles Gómez, Mariano Fernández, quienes estuvieron en todo momento apoyando el trabajo organizativo, así como al director titular del centro San Juan Bosco de Barcelona, don Ramón Vera, por acoger en su centro la celebración de estas jornadas que tan gratas sensaciones generaron en todos nosotros. Un agradecimiento especial fue dedicado al Dr. José Barrientos y a la asociación ETOR por su compromiso en la publicación de estas actas. Su apoyo y colaboración han sido indispensables para hacer posible este proyecto común.

La ceremonia de clausura finalizó con un power-point preparado por Humbert Ruiz sobre la frontera entre la demencia y la cordura, que, animado por un grupo de los asistentes portando máscaras, puso la nota final a tan entrañables días aportando un saludable toque de humor $y$ locura sana.

\section{Conclusiones}

El congreso de Barcelona permite afirmar que la filosofía aplicada en España goza, hoy por hoy, de una excelente buena salud. Ha evidenciado que en los últimos años la vitalidad de esta manera de entender la actividad filosófica ha ido in crescendo, de manera que en muchísimas ciudades de nuestro país, como Málaga, Zaragoza, Salamanca, Palma de Mallorca, Madrid, Valencia, Sevilla, Gerona, etc. el número de filósofos que suscriben la presencia de la filosofía en un buen número de espacios sociales en los que hasta ahora no se la esperaba resulta ya bien significativo.

Los cafés filosóficos están proliferando en centros cívicos, bibliotecas, cafeterías... En Gerona, el grupo Iref ofrece un Máster online 
de filosofía para niños desde hace años con un gran éxito, se hacen talleres filosóficos a distintos niveles, la lista de asesores filosóficos no cesa de aumentar en todas las comunidades, etc. No obstante, cabe reconocer que todavía queda mucho camino por recorrer. Siguen habiendo resistencias, y a veces muy fuertes, por parte de cierto sector de la academia, para aceptar que esta apertura de la filosofía hacia nuevos espacios sociales merezca su aprobación y respeto. Sin embargo, estamos convencidos de que la filosofía es demasiado valiosa para quedar recluida en los ámbitos de enseñanza, y que el futuro de la misma (así como de la propia academia, aunque ésta parezca no darse cuenta de ello) depende en buena medida del éxito de dicha apertura.De igual manera, el reconocimiento de la profesión de asesor filosófico y su normalización social andan aún muy lejos de estar establecidos. Los pasos que se están dando en esta dirección parecen ir por el buen camino, pero se nos antoja urgente que el colectivo de filósofos asesores sepa encontrar la manera de acompasar dichos pasos para poder generar las sinergias oportunas que permitan consolidar una voz común que reivindique el ejercicio de su labor profesional. De lo contrario, nos tememos que si cada uno camina por separado y no se aúnan esfuerzos por constituirse como un colectivo que reclama su presencia en los espacios sociales, las dificultades para que el movimiento de la filosofía aplicada goce del reconocimiento que todos deseamos podrían llegar a ser insalvables. Simplemente un apunte: en cuatro días todo el mundo ha oído hablar del PNL o del coaching. Sin embargo, la mayor parte de la población sigue desconociendo qué es eso del "asesoramiento filosófico". Hay que romper con la imagen del pensador en clave individualista, a lo Rodin, y tomar como referencia la danza de Matisse, pues como recoge la frase que no por ser muy tópica deja de seguir siendo válida: "la unión hace la fuerza".

\section{Para más información}

COTTA, SERGIO.: Las raíces de la violencia: una interpretación filosófica. Ediciones Universidad de Navarra, EUNSA, 1987.

COUPLAND, Douglash. Generación X. Tiempos modernos, Ediciones B, Bilbao, 1993. 
DUNNING, E.: El fenómeno deportivo: estudios sociológicos en torno al deporte, la violencia y la civilización, Paidotribo, Barcelona, 2003.

FERRY, L.: Vincent, J.D.. ¿Qué es el hombre? Sobre los fundamentos de la Biología y la Filosofía, Taurus, Madrid, 2001.

GARCÍA GÓMEZ - HERAS, J.M ${ }^{\mathrm{a}}$, Dignidad de la vida y manipulación genética: bioética, ingeniería genética, ética feminista, deontología médica. Editorial Biblioteca Nueva, D.L., Madrid, 2002.

HADOT, P.: Ejercicios espirituales y filosofía antigua. Ed. Siruela, Madrid, 2006.

HALL, R.: Bioética institucional. Problemas y prácticas en las organizaciones para el cuidado de la salud, Fontamara, México, 2008.

KREIMER, Roxana: "Prácticas filosóficas para cambiar la persona y la sociedad", en CAVALLÉ, Mónica y MACHADO, Julián D.: Arte de vivir, arte de pensar, Editorial Desclée de Brouwer, Bilbao, 2007.

MORRISON, A.: La cultura de la vergüenza, anatomía de un sentimiento ambiguo, Editorial Paidós, Barcelona, 1997.

NELSON, Leonard: El método socrático, el método regresivo, Huaqualya, Cádiz, 2008.

PAÑOSO, A. Filosofía del turismo. Teoría y epistemología. Ed. Trillas, S.A, México, 2008.

RUIZ GIL, H.: Del modelo cooperativo a la economía ética. IGOPAPOSTA, Barcelona, 2009.

SCHMID, W. En busca de un nuevo arte de vivir. Ed. Pre-textos, Valencia, 2002.

SHIPLEY, P., MASON, H. (Eds): Ethics and Socratic Dialogue in Civil Society, Lit Verlag, Münster, 2004.

TRÍAS, E.: El canto de las sirenas. Argumentos musicales. Ed. Galaxia Gutenberg, Barcelona, 2007.

WIJAYARATNA, M.: La philosophie du Bouddha, Lis, París, 2000. 
